\title{
Quicktime virtual reality technology in light microscopy to support medical education in pathology
}

\author{
Francesco Alfredo Zito ${ }^{1}$, Franco Marzullo ${ }^{1}$, Diego D’Errico ${ }^{1}$, Cesare Salvatore ${ }^{1}$, \\ Rosanna Digirolamo ${ }^{1}$, Angela Labriola ${ }^{1}$ and Antonio Pellecchia ${ }^{2}$ \\ ${ }^{1}$ Division of Pathology and ${ }^{2}$ Division of Digestive Endoscopy, National Cancer Institute, Bari, Italy
}

\begin{abstract}
The new computer-based interactive technologies in medicine, such as virtual reality (VR), have revolutionized education. The use of virtual microscopic images would be invaluable in the training of cyto-histopathologists. However, due to the vast amount of digital information on a scanned, conventional cyto-histological slide, which is enormous by current data storage standards, these systems are expensive and not widely used in pathological medicine. The authors propose an inexpensive system based on quicktime virtual reality (QTVR) technology (by Apple Computers Inc.), which accommodates a wide area of a slide at high magnification, generating a 'virtual slide' which makes it possible to navigate by conventional input devices. Commercial softwares that stitch consecutive, adjacent images of cyto-histological preparations onto a QTVR panorama were used. QTVR files have the ability to stand on their own as self-contained, multimedia applications and also have the ability to generate multinode scenes by means of 'hot spots'. QTVR 'movies' can be played on Macintosh or Windows platforms, and on major web browsers. Virtual slides by QTVR is an inexpensive system of high educational value, which allows the creation of multimedia databases of cyto-histological preparations that can exist on an internet server or can be distributed on removable media.
\end{abstract}

Modern Pathology (2004) 17, 728-731, advance online publication, 9 April 2004; doi:10.1038/modpathol.3800113

Keywords: quicktime VR; virtual reality; virtual slide; digital slide; telepathology; education

Virtual reality (VR) is a digital technology that allows people to interact with simulated computer environments using their natural senses. Its applications in medicine are increasing. ${ }^{1-12}$ Above all, VR plays an important role in medical training. In some medical fields, the educational value of simulation is probably superior to currently available methods of training. Although a wide range of VR training systems have been developed, at the moment, there are no systems available for cyto-histopathology that are efficient and cost effective, which can substitute for currently available educational methods. The reason for this difficulty is due to the fact that magnifying a single digitized histological slide requires a great deal of storage capacity. In the last few years, dynamic telepathology has been the only real digital application that has been able to transmit wide areas of microscopic preparations to a remote station. ${ }^{13-20}$ Dynamic telepathology is a real-time

Correspondence: FA Zito, IRCCS-Oncologico via Hahnemann 10, Bari 70126, Italy.

E-mail: istopatologia@oncologico.bari.it

Received 12 December 2003; revised 22 January 2004; accepted 23 January 2004; published online 9 April 2004 technology system, but its usefulness in training is limited. The image is live and only a few systems are able to store and create a database of the transmitted cases $^{18-20}$ (Bliss by BacusLabs. http://www.bacuslabs.com/). Furthermore, all of these systems are very expensive because they work by means of a robotic microscope, and the software for image viewing and interaction is often sophisticated and only available with the purchase of apparatus.

We propose an inexpensive system based on Quicktime VR (QTVR: trademarks used under license from Apple Computer) technology to digitize wide areas of cyto-histologic preparations by means of a software that generates a user friendly interface, allowing the viewer to navigate within a 'virtual slide' using conventional computer input devices, such as a mouse, trackball, trackpad, keyboard, etc. The software is not open source, but is a commercial product that uses the QTVR system, an extension of QuickTime technology, which is an industrial standard developed by Apple Computer Inc. for Macinthosh and Window platforms. The QTVR files may exist on an internet server and can be viewed by major browsers without the need of any additional client software. 


\section{Materials and methods}

A digital camera (Olympus 3030, 2.5 MB, Smart Media $128 \mathrm{MB}$ ) was mounted onto the microscope (Olympus $\mathrm{B} \times 41$ ) and connected to a computer (533 MHz Power Mac G4, 256 MB RAM, 22-inch monitor, system software Mac Os 9.2 and Mac Os X). Commercial softwares were used to manipulate the images (Camedia Master by Olympus; PhotoStitch by Canon; VR Worx 2.1 for Mac Os X by Toolbox; QuickTime 5 and QuickTime Player by Apple Comp. Inc.). The slide images were digitized to $2048 \times 1536$ pixels and saved in Joint Photography Expert Group format (JPEG) format.

\section{Image Acquisition}

Step 1: The images were acquired using the panorama option of the digital camera moving the

a

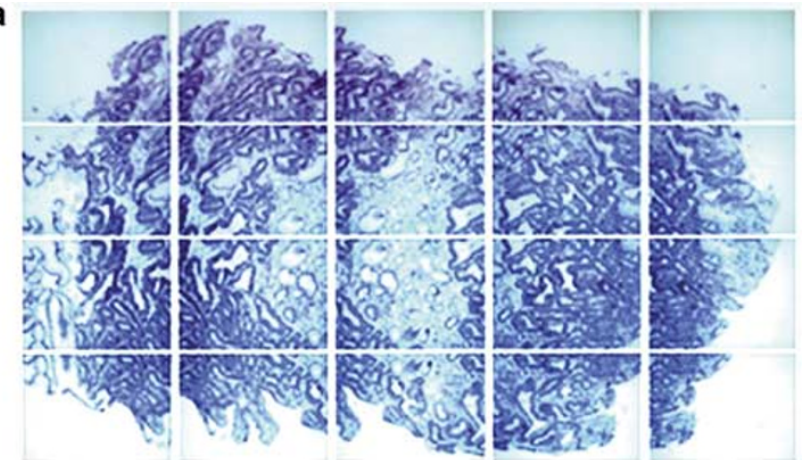

b

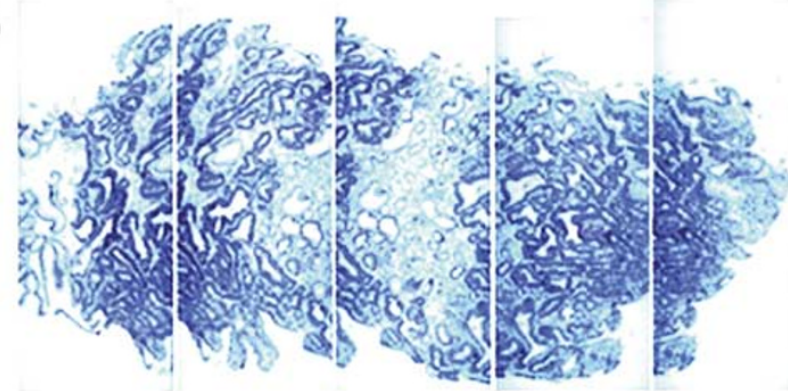

c

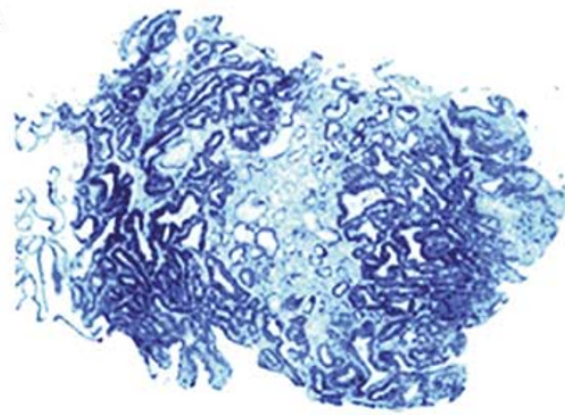

Figure 1 Composite figure showing the stitching sequence of a gastric adenocarcinoma biopsy fully digitized by a $\times 20$ objective. (a) Shows 20 partially overlapping still images. (b) Columns have been 'stitched' together. (c) Rows have been 'stitched' together (the image is $3 \%$ of the original). microscopic table along the ' $y$ '-axis so as to achieve about a $30 \%$ overlap of image tiles (Figure 1a).

Step 2: The stitching of the images was achieved by use of 'Camedia Master' free software bundled with the Olympus camera (http://cf.olympus-europa.com/consumer/digimg/home.cfm). Stitching of the images results in a series of parallel panoramic images, so as to achieve about $50 \%$ overlap with each other (Figure 1b).

Step 3: PhotoStitch software stitches the panoramic images together, generating a single large image of the area of interest (Figure 1c), and recorded in TIFF format.

Step 4: The image generated in step 3 was converted by VR Worx 2.1 software, resulting in a QTVR movie. The Photo JPEG codec (compressordecompressor) was selected to compress the QTVR 'movies' along with 24 bit (millions of colors) as the color depth chosen. QTVR format 'movies' can be viewed by QuickTime Player, a free application, already installed in many computers, which is available for Window and Macintosh platform.

\section{Results}

In order to replicate conventional microscopy, there is only one resizable window on the monitor. The viewer can navigate within the QTVR movie in all directions by clicking and dragging the mouse, or by using keyboard commands. The movie is linear and the keyboard is used to magnify or de-magnify the viewed area. More areas of interest can be connected to each other by 'hot spots' that are also used to perform certain actions, such as linking to other media files. 'Hot spots' allow the creation of scenes of multiple QTVR nodes as areas digitized to higher magnification, or differently stained (histochemical

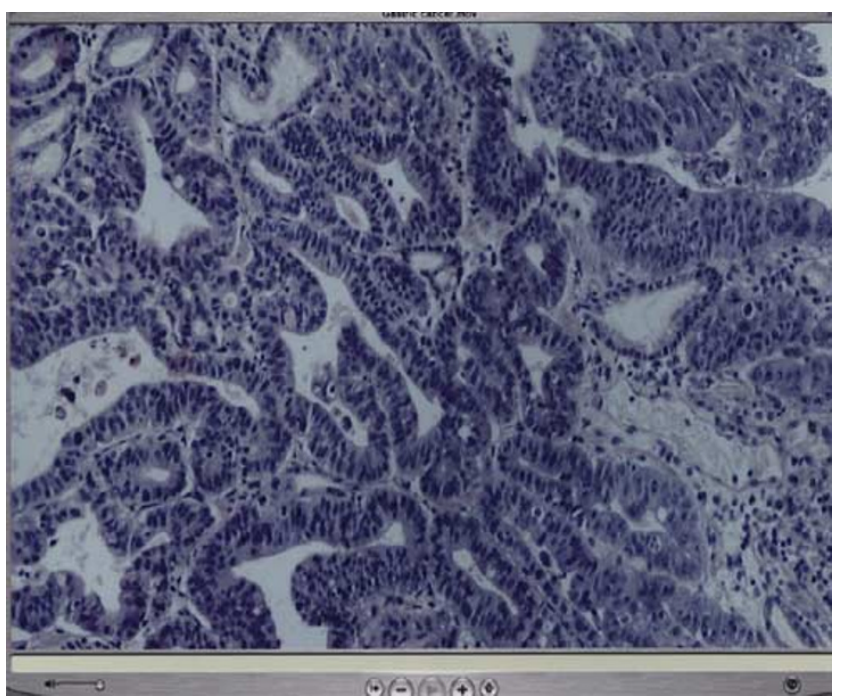

Figure 2 Window of the QuickTime Player showing a QTVR movie of a gastric adenocarcinoma, demonstrating the high quality of a single frame. 
or immunohistochemical stain), or they can refer to textual or audio annotations. QTVR format 'movies' can be viewed by Windows and Macintosh platforms. The system also allows the user to choose different compression formats (MPEG, JPEG, Sorenson etc). The optimal JPEG compression of QTVR files is $20 \%$ of the original TIFF file. These files can remain on the internet, or else can be stored on removable media (CD-ROM or DVD). The QTVR system works by merging the tiles around an imaginary cylinder. The effect is a spatial deformation of the tiles that can be reduced by selecting an angle of view less than $360^{\circ}$. Figure 1c shows a $3 \times 2 \mathrm{~mm}$ image of an endoscopic gastric biopsy. The $7200 \times 5200$ pixel image was digitized at an objective magnification of $\times 20$. A total of 20 still pictures are required to generate a large image of $85 \mathrm{MB}$ in TIFF format, which is then compressed into a $9 \mathrm{MB}$ QTVR file. The entire digitization process takes $60 \mathrm{~min}$. The quality of the movie is related to the quality of initial images (Figure 2).

\section{Discussion}

The new technologies, in particular VR, are being utilized more and more as instruments for learning in medicine. ${ }^{1-12}$ These systems, acting as medical simulators allow learning with absolute safety. This is obviously important in surgery or in invasive medical practice, but it is also important for other disciplines, as it offers students the ability to interact with instructional material in a way previously unimagined. The VR applications of pathological training should allow access to a large variety of cyto-histological cases. However, terms such as 'virtual histology', 'digital slide' and 'virtual microscopy', which appear in the literature, referring to some digital applications viewing cyto-histologic preparations have been improperly used for two reasons. Firstly, because these often refer to static images, and secondly because the software generated an interface completely extraneous to the way in which the pathologist works. With our system, the observer can perceive himself in the surrounding environment. For a system to truly be called 'VR', it must be able to interact with the user in a precise and immediate manner. A virtual slide, therefore, must have the same capacity. With each movement of the cursor, the computer must instantaneously recalculate the new position of the observer in the virtual environment. A 'QTVR slide' accomplishes this. The major advantage is that the case is not a selected, static image, but a 'real virtual slide' in which the observer can navigate and choose specific areas, magnifying and de-magnifying as he/ she wishes, allowing learning to occur in a way similar to conventional microscopy. Trelease et $a l^{1}$ were the first to propose the application of QTVR technology in light microscopy to support instruction and research in microscopic anatomy, generat- ing histological panoramic movies with the capacity to be integrated into existing web-based resources for use in a first-year medical histology course at UCLA School of Medicine, Los Angeles. The use of such technology in the field of pathology would have much broader implications. Recently, MelinAldana and Sciortino ${ }^{2}$ have used QTVR in pathology primarily to generate three-dimensional reconstructions of surgical specimens. The authors of this article propose the use of the same technology in pathology whereby it is possible to view a large portion of a cyto-histological slide in order to make accurate diagnoses, as well as for use in other telepathology applications, including training of students, staff, etc. Virtual slides also allow one to overcome some difficulties in pathological practice. For example, conventional glass histology slides are fragile, and stains can fade with time, as is the case with some staining techniques such as immunofluorescence. Another difficulty is that distribution of glass slides among pathologists during quality assurance programs are not the most practical way to gather information for statistical assessment due the significant differences that can occur when cutting multiple tissue sections of the same paraffin block, creating a 'misrepresentation' of the tissue sample. In addition, the participation of cytopathologists during quality assurance must currently be limited to small groups because it is not possible to cut sections of the same slide, and therefore requires much more time to circulate a single set of glass slides. Complete slide digitization of high magnification would be the goal that provides a solution. Unfortunately, the volume of digitized data on a whole slide is so big that it requires complex and expensive systems. Leong and McGee ${ }^{18}$ have calculated that digitizing a $10 \times 10 \mathrm{~mm}$ region of solid tumor using a $\times 20$ objective lens generates about 2.75 gigabytes (GB) of raw image data that falls to $250 \mathrm{MB}$ by JPEG compression. They used a robotic microscope that takes $70 \mathrm{~min}$ to digitize $1 \mathrm{~cm}^{2}$ of slide. The time, the high cost of hardware and magnetic support of storage required have discouraged wide use of these systems. As with any new technology, cost is a major consideration. Our system is inexpensive because it works with common microscopic equipment connected to a nonprofessional digital camera. Viewing of the histological 'movie' is obtained by use of commercial software. The cost of our nonautomated system, excluding microscope, is less than $\$ 6000$ in comparison to the \$70000-90000 cost of motorized systems. It is important to note however, that manually digitizing a large area of slide by an objective magnification of at least $\times 20$ is very difficult due to the high number of individual images required. Therefore, it is preferable to use this system on small biopsies. The possibility to create 'hot spots' allows us to navigate whole slides, which can be linked to other files or URL, generating a digital medium with a high educational value. Histological slide files are 
in QTVR compressed format that can be stored on CD-ROMs or in a database on a server. The latter can be used to download the case library with a common browser connected to the internet.

The most important telepathology systems work by means of an overview image of low magnification of the entire slide, displaying the current position of the observer, choosing the area of interest that the viewer wants to magnify. This system requires at least two windows to be open on the monitor, one of the windows being the overview. The other window may be the selected area at a higher magnification. Selecting an image to be magnified is not very practical because it constrains the user to pass from one window to another. Our system requires only one open window on the monitor. Movement through the virtual slide is continuous and the magnification and demagnification is obtained by pressing a combination of keys (shift/ctrl). As a result, a monitor with a large display is not necessary.

There are other advantages in using QTVR movies: They are self-contained; they have the ability to be integrated into other various multimedia programs, producing complete instructional tutorials and research presentations; and paradoxically, having all the options available that QTVR movies offer is much more difficult to find in other, more expensive, and more complex systems.

In conclusion, our system is simple and inexpensive. And although the emphasis is chiefly on teaching medical students and on the continuing education of physicians, it also has the capacity to be used in other common telepathology applications.

\section{Acknowledgement}

We thank Michael Kolk for his help in the preparation of this manuscript.

\section{References}

1 Trelease RB, Nieder GL, Dorup J, et al. Going virtual with QuickTime VR: new methods and standardized tools for interactive dynamic visualization of anatomical structures. Anat Rec 2000;261:64-77.

2 Melin-Aldana H, Sciortino D. Virtual reality demonstration of surgical specimens, including links to histologic features. Mod Pathol 2003;16:958-961.

3 Erel E, Aiyenibe B, Butler PE. Microsurgery simulators in virtual reality: review. Microsurgery 2003;23: 147-152.
4 Gorman PJ, Meier AH, Rawn C, et al. The future of medical education is no longer blood and guts, it is bits and bytes. Am J Surg 2000;180:353-356.

5 Engum SA, Jeffries P, Fisher L. Intravenous catheter training system: computer-based education versus traditional learning methods. Am J Surg 2003;186: 67-74.

6 Gerson LB, Van Dam J. A prospective randomized trial comparing a virtual reality simulator to bedside teaching for training in sigmoidoscopy. Endoscopy 2003;35:569-575.

7 Schultheis MT, Himelstein J, Rizzo AA. Virtual reality and neuropsychology: upgrading the current tools. J Head Trauma Rehabil 2002;17:378-394.

8 Dobson HD, Pearl RK, Orsay CP, et al. Virtual reality: new method of teaching anorectal and pelvic floor anatomy. Dis Colon Rectum 2003;46:349-352.

9 Bloom MB, Rawn CL, Salzberg AD, et al. Virtual reality applied to procedural testing: the next era. Ann Surg 2003;237:442-448.

10 Spicer MA, Apuzzo ML. Virtual reality surgery: neurosurgery and the contemporary landscape. Neurosurgery 2003;52:489-497.

11 Verma D, Wills D, Verma M. Virtual reality simulator for vitreoretinal surgery. Eye 2003;17:71-73.

12 McCloy R, Stone R. Virtual reality in surgery. BMJ 2001;323:912-915.

13 Demichelis F, Barbareschi M, Boi S, et al. Robotic telepathology for intraoperative remote diagnosis using a still-imaging-based system. Am J Clin Pathol 2001;116:744-752.

14 Kaplan KJ, Burgess JR, Sandberg GD, et al. Use of robotic telepathology for frozen-section diagnosis: a retrospective trial of a telepathology system for intraoperative consultation. Mod Pathol 2002;15: 1197-1204.

15 Dunn BE, Choi H, Almagro UA, et al. Combined robotic and nonrobotic telepathology as an integral service component of a geographically dispersed laboratory network. Hum Pathol 2001;32:1300-1303.

16 Wellnitz U, Fritz P, Voudouri V, et al. The validity of telepathological frozen section diagnosis with ISDNmediated remote microscopy. Virchows Arch 2000; 437:52-57.

17 Weisz-Carrington $\mathrm{P}$, Blount $\mathrm{M}$, Kipreos B, et al. Telepathology between Richmond and Beckley Veterans Affairs Hospitals: report on the first 1000 cases. Telemed J 1999;5:367-373.

18 Leong FJ, McGee JO. Automated complete slide digitization: a medium for simultaneous viewing by multiple pathologists. J Pathol 2001;195:508-514.

19 Demichelis F, Barbareschi M, Dalla Palma P, et al. The virtual case: a new method to completely digitize cytological and histological slides. Virchows Arch 2002;441:159-164.

20 Demichelis F, Della Mea V, Forti S, et al. Digital storage of glass slides for quality assurance in histopathology and cytopathology. J Telemed Telecare 2002;8: 138-142. 